\title{
Advancing our diagnostic tools and treatment options for delirium
}

Delirium is one of the most prevalent and disabling conditions impacting older adults in hospitals and long-term care settings. The estimates of frequency of delirium in hospitals and nursing homes among older adults range widely, yet it is safe to assume that perhaps as many as $25-30 \%$ of elderly individuals in hospitals experience an episode of delirium, often undetected unless a regular screening process is implemented. Until the past couple of decades, actual research into delirium had been sparse and done little to inform clinical practice. Engel and Romano (1959) published a classic review nearly 60 years ago, which served for decades as the foundational article for understanding delirium. In recent years, however, our understanding of delirium has increased dramatically, from the basic neuropathological underpinnings through screening and diagnosis to treatment. In this issue of International Psychogeriatrics, five papers are published, which further expand our knowledge of delirium. The papers cover a range of topics including the frequency of delirium in a primary care setting, outcomes predicted by various diagnostic systems, cognitive function measures as they relate to delirium motor subtypes, screening for delirium using a standardized drug screen, and the effectiveness of multimodal interventions for preventing delirium in older hip fracture patients. What are the take home lessons from these studies?

Bohlken and Kostev (2018) compared persons with and without delirium, who were $65+$ years of age with over 2,000 delirium and 2,000 controls derived from an administrative data base of over $6,000,000$ general practice participants from Germany. The prevalence of delirium is extremely low in these outpatient settings, and this is not a surprise, even though some nursing home participants were included in the sample. At least two considerations should be kept in mind regarding this low prevalence. First, the authors used a data base with ICD diagnoses. In other words, there were no reported screening instruments routinely administered, such as the Confusion Assessment Method (CAM) (Inouye et al., 1990). To be fair, however, the low prevalence of delirium in primary care outpatient settings does not suggest a need for screening all patients for delirium. It would make much more sense, if screening were implemented, to screen for much more prevalent conditions, such as depression or substance use disorders. Second, delirium is basically a hospital phenomenon, either with patients admitted for delirium or developing delirium while hospitalized. Risk factors for delirium in this study included most of the usual suspects: dementia, benzodiazepines, sleep disorder, and residence in a nursing home. The take home message is that delirium will occasionally be found in outpatient settings though it is quite rare. The manifestations and the risks are no different than found in the hospital, except for hospital specific risks (such as surgery).

Adamis et al. (2018) compare outcomes for four diagnostic systems: DSM-5, DSM-IV, CAM, and DRS-R98. It should be noted from the outset that the CAM and DRS-R98 are actually screening instruments with specific probes, whereas DSM5 and DSM-IV list diagnostic criteria that can be applied in making a diagnosis of delirium. Even so, the comparison is of clinical interest. The frequency of delirium was nearly identical across the diagnostic systems and instruments, except for a slightly lower frequency on the DRS-R98. A total of 32 of the 68 cases of delirium, identified by one of the instruments, were identified by 3 of the 4 approaches. DSM-IV was the outlier, identifying 12 cases not identified otherwise. Mortality was predicted by all instruments, but variation occurred with other outcome measures. The take home message is that the case finding will vary according to the approach taken and the outcomes will vary according to which cases are identified by which method. Therefore, the clinicians should choose a method for identifying delirium that fits their clinical needs. Perhaps a combination of a screening instrument, such as the CAM, and a diagnostic system, such as DSM-5, would be the ideal approach.

Daly et al. (2018) examined hospital participants with full syndromal delirium, subsyndromal delirium, and no delirium using the DRS-R98 and an informant questionnaire on cognitive decline. Overall ability to participate in tests for attention, 
vigilance, and visuospatial function was fair but not exceptional with the best ability to perform found with the Spatial Span Forward Test. The authors note the problem of evaluating delirium participants in the hospital given their inability to participate in the evaluation. Of most interest is the categorization of delirium participants into four motor subtypes: none, hyperactive, mixed, and hypoactive. Many clinicians do not distinguish the motor subtypes, though DSM-5 does call upon clinicians to specify them in the diagnostic process. When no motor subtype is identified, participants performed better on all the neurocognitive tests and experienced a less severe delirium. Performance was similar for the hyperactive and hypoactive subtypes. The take home message from this study is twofold. First, though we have some excellent screening instruments, such as the CAM and the TRS-R98, to explore the intricacies of cognition in delirium, we must delve further and develop instruments that can both be administered with minimal difficulty to delirious participants and that can provide additional data for understanding underlying neurocognition, which can then inform treatment. Second, we must pay closer attention to motor subtypes when we first diagnose delirium and use these subtypes to help us predict outcomes and manage patients appropriately.

Nguyen et al. screened over 1,000 participants in three emergency departments (EDs) in Canada. Participants were on average $80+$ years old. They administered the Delirium Drug Scale (DDS), a scale that assesses drugs known to be associated with delirium. The diagnosis was based upon clinical diagnoses coupled with a review of key words in the written record, which might identify delirium. The frequency of delirium was $19.1 \%$. Investigators found that $62 \%$ of the participants were exposed to a DDS medication, the most frequent being benzodiazepines. If more medications were prescribed, the likelihood of delirium had been greater, even in controlled analysis. As clinicians know, medications are a prime cause of delirium as demonstrated in this study. The take home message from this study is twofold. First, the ED physician must always be aware that medications are one of the prime, if not the prime suspect for causing delirium in emergency situations. Second, these clinicians must be aware of the drugs that are likely to cause delirium, namely those evaluated by the DDS.

Oberai et al. reviewed nine studies (three were randomized controlled trials [RCTs]) that evaluated multimodal interventions for the prevention of delirium in hospitalized older adults with hip fracture. The nine studies, when combined, included nearly 1,900 participants. Overall, the incidence of delirium in the intervention groups was significantly reduced, and in the RCTs, the duration of delirium, if it occurred, was reduced as well. This review adds to the evidence that delirium can be partially prevented (though not eliminated) through efforts that go beyond the simple elimination of medications known to potentially cause delirium. Other components of multimodal intervention include addressing sensory impairment, frequent efforts to orient patients, assuring adequate bowel and bladder function, optimizing adequate sleep, and the treatment of medical complications. The take home message from this study, not a new one but one that cannot be emphasized enough, is that a comprehensive plan for delirium prevention in at-risk older adults (and elderly individuals who undergo surgery for hip fracture are at significant risk) is much more effective than a simple medication screen. Unfortunately, hospitals have not been quick to uptake the multimodal methods.

We have come a long way in our understanding of delirium during the past 20 years. And, the future looks bright for our understanding of delirium's neuropathological underpinnings, its neurocognitive deficits, its diagnosis, and its treatment.

\section{Conflict of interest}

None.

\section{DAN G. BLAZER}

Duke University Medical Center, Box 3003, Durham, NC 27710, USA

Email: dan.g.blazer@duke.edu

\section{References}

Adamis, D., Meagher, D., Rooney, S., Mulligan, O. and McCarthy, G. (2018). A comparison of outcomes according to different diagnostic systems for delirium (DSM-5, DSM-IV, CAM, and DRS-R98). International Psychogeriatrics, 30, 591-596.

Bohlken, J. and Kostev, K. (2018). Prevalence and risk factors for delirium diagnosis in patients followed in general practices in Germany. International Psychogeriatrics, 30, 511-518.

Daly, C. et al. (2018). Attention, vigilance, and visuospatial function in hospitalized elderly medical patients: relationship to delirium syndromal status and motor subtype profile. International Psychogeriatrics, 30, 493-501.

Engel, G. L. and Romano, J. (1959). Delirium: a syndrome of cerebral insufficiency. Fournal of Chronic Diseases, 9, 260-277. 
Inouye, S. K., vanDyck, C. H., Alessi, C. A., Balkin, S., Siegal, A. P. and Horwitz, R. I. (1990). Clarifying confusion: the confusion assessment method. A new method for detection of delirium. Annals of Internal Medicine, 113, 941-948.

Nguyen, P. V., Pelletier, L., Payot, I. and Latour, J. The delirium drug scale is associated to delirium incidence in the emergency department. International Psychogeriatrics, 30, 503-510.

Oberai, T., Laver, K., Crotty, M., Killington, M. and Jaarsma, R. Effectiveness of multicomponent interventions on incidence of delirium in hospitalized older patients with hip fracture: a systematic review. International Psychogeriatrics, 30, 481-492. 